\title{
Investigating the possible connection between $\lambda$ Bootis stars and intermediate Population II type stars
}

\author{
E. Paunzen ${ }^{1}$, I. Kh. Iliev ${ }^{2}$, L. Fossati ${ }^{3}$, U. Heiter ${ }^{4}$, and W. W. Weiss ${ }^{5}$ \\ ${ }^{1}$ Department of Theoretical Physics and Astrophysics, Masaryk University, Kotlářská 2, 61137 Brno, Czech Republic \\ e-mail: epaunzen@physics.muni.cz \\ 2 Rozhen National Astronomical Observatory, Institute of Astronomy of the Bulgarian Academy of Sciences, PO Box 136, \\ 4700 Smolyan, Bulgaria \\ 3 Argelander-Institut für Astronomie der Universität Bonn, Auf dem Hügel 71, 53121 Bonn, Germany \\ 4 Institutionen för fysik och astronomi, Uppsala universitet, Box 516, 75120 Uppsala, Sweden \\ 5 Institute for Astrophysics, University of Vienna, Türkenschanzstr. 17, 1180 Vienna, Austria
}

Received 16 March 2014 / Accepted 2 June 2014

\begin{abstract}
Context. The $\lambda$ Bootis stars are located at the upper main sequence of the Hertzsprung-Russell diagram and exhibit a peculiar abundance pattern. The light elements $(\mathrm{C}, \mathrm{N}, \mathrm{O}$, and $\mathrm{S})$ present solar abundances whereas all other elements are moderately to strongly underabundant. It has not yet been determined whether that abundance pattern is intrinsic, or is restricted to the stellar surface.

Aims. If we follow the hypothesis that the $\lambda$ Bootis stars are intrinsically metal-weak, then there should be a connection with the intermediate Population II and F-weak objects. Such a possible affinity has not been previously investigated.

Methods. We present detailed elemental abundances, including those of the light elements carbon and oxygen, for 38 bright intermediate Population II and F-weak objects. In addition, we investigate the kinematic characteristics of the groups.

Results. From photometric, spectroscopic, and kinematic data, there is no distinction between the intermediate Population II and F-weak type stars. We therefore conclude that the two groups are identical. However, it is possible to distinguish the $\lambda$ Bootis stars from the intermediate Population II stars on the basis of elemental abundances, though not in terms of their kinematics.

Conclusions. The $\lambda$ Bootis stars seem to be distinct from the intermediate Population II group. Further asteroseismologic investigations and analyses of spectroscopic binary systems are needed to strengthen this conclusion.
\end{abstract}

Key words. stars: abundances - stars: chemically peculiar - stars: early-type - stars: Population II

\section{Introduction}

The $\lambda$ Bootis stars are a small group (only $2 \%$ ) of late-B to early$\mathrm{F}$ stars that show moderate to extreme surface underabundances (up to a factor of 100) of most Fe-peak elements, but solar abundances of lighter elements $(\mathrm{C}, \mathrm{N}, \mathrm{O}$, and $\mathrm{S})$. They stand out among the chemically peculiar stars of the upper main sequence because all other groups exhibit strong overabundances of elements, probably caused by magnetic fields, slow rotation or atmospheric diffusion (e.g. Netopil et al. 2008). Several members of the group exhibit a strong infrared excess and a disk (Paunzen et al. 2003; Booth et al. 2013).

To explain the $\lambda$ Bootis phenomenon, Venn \& Lambert (1990) suggested that the peculiar chemical abundance is caused by selective accretion of circumstellar material. One of the principal features of that hypothesis is that the observed abundance anomalies are restricted to the stellar surface. Members of the group are therefore true Population I type objects. Later, Kamp \& Paunzen (2002) and Martinez-Galarza et al. (2009) developed models that describe the interaction of the star with its local interstellar and/or circumstellar environment, whereby different degrees of underabundance are produced by different amounts of accreted material relative to the photospheric mass. The fact that the fraction of $\lambda$ Bootis stars on the main sequence is so small would then be a consequence of the low probability of a star-cloud interaction, and by the effects of meridional circulation which dissolves any accretion pattern a few million years after the accretion has stopped. The hot cut-off would be the limitation of significant stellar winds for stars with $T_{\text {eff }}>12000 \mathrm{~K}$ whereas the cool end, at about $6500 \mathrm{~K}$, would be defined by convection which prevents the accreted material from appearing at the stellar surface.

The important question as to whether or not $\lambda$ Bootis stars are intrinsically metal-weak does not seem to have been raised very often in the literature. Recently, Casas et al. (2009) made a detailed asteroseismological analysis of the pulsational behaviour of 29 Cygni (HR 7736), which is one of the prototypes of the $\lambda$ Bootis group and exhibits very strong underabundances of the Fe-peak elements compared to the $\operatorname{Sun}([\mathrm{Fe} / \mathrm{H}]=-1.63 \mathrm{dex}$; Paunzen et al. 2002). From their best-fitting models they concluded that $29 \mathrm{Cyg}$ is an intrinsic metal-weak main-sequence object. Unfortunately, there is only one other similar analysis in the literature: Breger et al. (2006) investigated the pulsational characteristics of HD 210111 (HR 8437) and found no differences from the behaviour of the classical $\delta$ Scuti star, FG Virginis, which has a similar evolutionary status. The star HD 210111 was later found to be a spectroscopic binary system with almost equal components (Paunzen et al. 2012). Although the components have $[\mathrm{M} / \mathrm{H}]=-1.0 \mathrm{dex}$, the pulsational models of Breger et al. (2006) adopted solar abundances. It would be very interesting 
Table 1. Astrophysical and kinematic parameters for our target sample.

\begin{tabular}{|c|c|c|c|c|c|c|c|c|c|c|}
\hline HD & HIP & $\begin{array}{c}V \\
{[\mathrm{mag}]}\end{array}$ & $\begin{array}{l}T_{\text {eff }} \\
{[\mathrm{K}]}\end{array}$ & $\begin{array}{l}\log g \\
\text { [dex] }\end{array}$ & $\begin{array}{c}M_{\mathrm{V}} \\
{[\mathrm{mag}]}\end{array}$ & $\begin{array}{c}v \sin i \\
{\left[\mathrm{~km} \mathrm{~s}^{-1}\right]}\end{array}$ & $\begin{array}{c}R_{\mathrm{V}} \\
{\left[\mathrm{km} \mathrm{s}^{-1}\right]}\end{array}$ & $\begin{array}{c}U \\
{\left[\mathrm{~km} \mathrm{~s}^{-1}\right]}\end{array}$ & $\begin{array}{c}V \\
{\left[\mathrm{~km} \mathrm{~s}^{-1}\right]}\end{array}$ & $\begin{array}{c}W \\
{\left[\mathrm{~km} \mathrm{~s}^{-1}\right]}\end{array}$ \\
\hline 693 & 910 & 4.91 & $6200(55)$ & $4.10(7)$ & $3.53(2)$ & $6.0(3)$ & $+13.5(4.1)$ & $+18.9(2)$ & $-13.4(1.1)$ & $-16.8(4.0)$ \\
\hline 3229 & 2787 & 5.94 & $6350(88)$ & $3.80(15)$ & $2.22(5)$ & $6.0(6)$ & $+6.2(2.5)$ & $-25.5(9)$ & $-29.2(1.1)$ & $-15.2(2.3)$ \\
\hline 4813 & 3909 & 5.18 & $6150(34)$ & $4.35(11)$ & $4.23(2)$ & $5.4(3)$ & $+9.3(2.2)$ & $+21.2(4)$ & $-2.3(6)$ & $-13.6(2.1)$ \\
\hline 7476 & 5833 & 5.70 & $6450(50)$ & $3.90(15)$ & $2.48(6)$ & $6.8(1)$ & $+25.5(2.5)$ & $-26.7(1.0)$ & $+41.9(1.2)$ & $-4.2(2.3)$ \\
\hline 9919 & 7535 & 5.60 & $6850(49)$ & $4.00(5)$ & $2.94(7)$ & $105.9(7.8)$ & $-1.0(2.5)$ & $+9.2(1.3)$ & $+8.0(1.1)$ & $-0.4(1.9)$ \\
\hline 12414 & 9473 & 7.06 & $6250(47)$ & $4.25(15)$ & $3.72(5)$ & $6.0(9)$ & $+14.1(2.5)$ & $-22.7(1.4)$ & $-20.5(9)$ & $-9.9(2.0)$ \\
\hline 13555 & 10306 & 5.23 & $6450(85)$ & $4.15(11)$ & $2.80(5)$ & 7.4(4) & $+6.4(6)$ & $-20.7(6)$ & $-11.8(5)$ & $+3.7(4)$ \\
\hline 16626 & 12656 & 7.02 & $6550(99)$ & $4.20(15)$ & $3.32(8)$ & $17.0(2.5)$ & $+14.9(2.5)$ & $-17.6(1.8)$ & $+3.8(1.8)$ & $-13.5(6)$ \\
\hline 17948 & 13665 & 5.59 & $6450(65)$ & $4.20(8)$ & $3.48(3)$ & $9.0(1$ & $+29.2(2.5)$ & $-30.9(1.8)$ & $+9.0(1.7)$ & $+12.9(2)$ \\
\hline 24740 & 18471 & 5.70 & $6900(81)$ & $3.75(1)$ & $2.42(8)$ & $21.0(2.1)$ & $+32.2(2.5)$ & $-31.4(2.2)$ & $-22.3(8)$ & $-19.2(1.3)$ \\
\hline 25457 & 18859 & 5.36 & $6250(59)$ & $4.45(24)$ & $3.94(4)$ & $19.2(1.2)$ & $+18.1(8)$ & $-8.3(6)$ & $-28.8(4)$ & $-12.2(6)$ \\
\hline 26015 & 19261 & 6.02 & $6750(43)$ & $4.15(12)$ & $2.62(9)$ & $30.0(4.5)$ & $+37.7(2.7)$ & $-42.7(2.4)$ & $-20.6(8)$ & $-0.7(1.5)$ \\
\hline 27397 & 20219 & 5.58 & $7300(57)$ & $3.95(5)$ & $2.30(8)$ & $100.0(5.8)$ & $+42.0(2.5)$ & $-44.8(2.3)$ & $-19.2(6)$ & $-2.8(1.3)$ \\
\hline 27524 & 20349 & 6.79 & $6550(87)$ & 4.3 & $3.22(10)$ & 90.0 & +37.1 & -4 & - & $-0.1(1.3)$ \\
\hline 27808 & 20557 & 7.13 & $6200(61)$ & $4.35(21)$ & $4.05(8)$ & 12.0 & +36.8 & -40.4 & $-18.4(7)$ & $-1.2(1.2)$ \\
\hline 29391 & 21547 & 5.22 & $7300(73)$ & $4.20(7)$ & $2.84(4)$ & $77.9(1.8)$ & $+21.0(2.5)$ & $-14.0(2.0)$ & $-16.2(7)$ & $-10.1(1.3)$ \\
\hline 33256 & 23941 & 5.12 & $6350(80)$ & $3.95(21)$ & $3.11(4)$ & $9.9(6)$ & $+10.2(1.2)$ & $-10.4(1.0)$ & $-6.3(5)$ & $+1.8(5)$ \\
\hline 65123 & 38870 & 6.35 & $6300(41)$ & $4.25(17)$ & $2.44(13)$ & $13.0(2.0)$ & $+1.1(2.1)$ & $-22.9(2.0)$ & $+9.7(1.5)$ & $-38.3(2.4)$ \\
\hline 69897 & 40843 & 5.15 & $6300(47)$ & $4.35(8)$ & $3.85(3)$ & $4.2(2)$ & $+32.8(5)$ & $-24.3(4)$ & $-38.5(5)$ & $+7.2(3)$ \\
\hline 110093 & 61396 & 7.23 & $7450(36)$ & $4.00(15)$ & $2.48(9)$ & $115.0(20)$ & $-12.0(2.5)$ & $-7.4(1.3)$ & $-13.0(1.8)$ & $-11.9(1.4)$ \\
\hline 114642 & 64407 & 5.03 & $6350(59)$ & $3.90(16)$ & $2.46(3)$ & $13.5(6)$ & $-14.1(2.5)$ & $+22.4(1.2)$ & $-11.7(1.4)$ & $-41.8(1.8)$ \\
\hline 125111 & 69735 & 6.20 & $6700(72)$ & $4.15(1)$ & $2.78(6)$ & $9.5(3)$ & $-25.0(1.2)$ & $-25.9(6)$ & $-28.5(7)$ & $-13.2(1.2)$ \\
\hline 125276 & 69965 & 5.87 & $6100(88)$ & $4.55(18)$ & $4.62(3)$ & $3.9(3)$ & $-21.0(2.5)$ & $-41.5(1.8)$ & $+9.0(1.3)$ & $+22.3(1.4)$ \\
\hline 128167 & 71284 & 4.47 & $6750(61)$ & $4.30(4)$ & $3.52(2)$ & $9.5(1)$ & $+0.2(2)$ & $+2.1(1)$ & $+15.9(1)$ & $-5.2(2)$ \\
\hline 135208 & 74593 & 6.77 & 6450(79) & $3.85(15)$ & $2.58(9)$ & $9.0(1$ & $-24.9(7)$ & $-36.7(9)$ & -21 & $-0.3(1.0)$ \\
\hline 006 & 75342 & 6 & $7450(62)$ & $4.05(6)$ & (9) & 127. & -2.2 & +12.0 & $+7.7(6)$ & -15 \\
\hline 147449 & 80179 & 4.83 & 7000(49) & $4.05(5)$ & $2.63(6)$ & 77.7(6) & $-49.8(2.5)$ & $-49.7(2.1)$ & $-18.8(6)$ & $-10.2(1.4)$ \\
\hline 157950 & 85365 & 4.53 & $6700(43)$ & $4.00(69$ & $2.13(4)$ & $27.0(3)$ & $-0.1(9)$ & $+1.4(8)$ & $-12.0(4)$ & $+7.9(4)$ \\
\hline 185124 & 96556 & 5.45 & $6650(43)$ & $4.15(19)$ & $2.88(3)$ & $84.3(6.1)$ & $-37.6(2.5)$ & $-33.6(2.0)$ & $-22.8(1.4)$ & $-9.4(6)$ \\
\hline 189712 & 98565 & 7.46 & $6450(120)$ & $3.85(15)$ & $2.85(13)$ & $8.0(1.2)$ & $+31.5(2.5)$ & $+32.0(1.7)$ & $+13.9(1.9)$ & $-0.6(9)$ \\
\hline 191841 & 99558 & 7.33 & $6450(72)$ & $4.25(10)$ & $2.72(15)$ & $14.0(2.1)$ & $+6.6(2.5)$ & $+9.8(2.0)$ & $-7.9(1.6)$ & $-9.0(1.1)$ \\
\hline 198390 & 102805 & 5.90 & $6500(75)$ & $4.30(11)$ & $3.51(3)$ & $6.7(2)$ & $+2.3(2.5)$ & $-11.9(1.2)$ & $+10.4(2.0)$ & $+0.8(8)$ \\
\hline 199124 & 103261 & 6.56 & $7200(58)$ & $3.80(9)$ & $2.03(9)$ & $159.1(11.5)$ & $-6.6(4.0)$ & $-11.7(2.4)$ & $+3.2(2.6)$ & $+3.4(1.9)$ \\
\hline 208362 & 108147 & 7.46 & 6750(116) & $4.15(15)$ & $3.20(10)$ & $17.9(1.4)$ & $+20.6(4.0)$ & $-21.3(7)$ & $+19.5(4.0)$ & $+3.7(8)$ \\
\hline 211575 & 110091 & 6.40 & $6500(39)$ & $4.15(13)$ & $3.31(5)$ & $19.0(1.6)$ & $+15.8(1.4)$ & $+17.0(5)$ & $+3.5(1.0)$ & $-11.4(1.0)$ \\
\hline 218804 & 114430 & 6.00 & $6400(81)$ & $4.10(9)$ & $3.73(3)$ & 17.2(9) & $-43.4(2.5)$ & $+46.0(7)$ & $-35.6(2.3)$ & $-2.6(7)$ \\
\hline 222368 & 116771 & 4.13 & $6200(36)$ & $4.15(15)$ & $3.43(2)$ & $6.3(1)$ & $+4.1(3.5)$ & $-7.8(3)$ & $-27.1(2.1)$ & $-25.3(2.8)$ \\
\hline 223346 & 117445 & 6.47 & $6350(71)$ & $3.90(13)$ & $2.76(7)$ & $18.9(1.4)$ & $-25.0(2.5)$ & $+3.2(4)$ & $-21.3(1.4)$ & $+15.9(2.1)$ \\
\hline
\end{tabular}

Notes. The uncertainties in the final digits of the corresponding quantity are given in parentheses.

to re-analyse their observations with corresponding metal-weak models.

Incorporating unrepresentative metal abundances in the models has consequences not only for the theories already developed to explain the $\lambda$ Bootis phenomenon, but also for the calibration of the astrophysical parameters. For example, in order to derive stellar ages one would need to use isochrones calculated for abundances that were intrinsically non-solar.

As a rider to that hypothesis, it would be difficult to describe the $\lambda$ Boo stars as true Population I type objects. A first possible connection between these star groups was suggested by Gray (1989), who introduced a new classification scheme for the intermediate Population II (hereafter ip II) stars. In this paper we present a fresh detailed investigation of a possible connection between the F-weak objects and ip II ones, using detailed elemental abundances, photometric diagrams, and kinematic data.

\section{Target selection, observations, and reductions}

We chose 38 bright $(V<7.5 \mathrm{mag}$ ) F-weak and ip II stars from the samples by Jaschek et al. (1989) and Gray (1989); all have parallaxes measured by HIPPARcos (van Leeuwen 2009). We expressly selected objects that overlap the $\lambda$ Bootis stars in terms of effective temperature and surface gravity. The stars are listed in Table 1.

Observations of the sample were made between 2001-2002 with the coudé spectrograph of the $2 \mathrm{~m}$ Ritchey-Chrétien telescope of the Bulgarian National Astronomical Observatory, Rozhen, using the Photometrics AT200 camera with a SITe SI003AB $1024 \times 1024$ CCD chip. Typical seeing was 2-3"; the slit width was set to $300 \mu \mathrm{m}$, which corresponded to 0 .' $^{\prime}$ on the sky. A Bausch \& Lomb grating with 632 lines $\mathrm{mm}^{-1}$ was centred on two different spectral regions, $7120 \AA$ (C-region) and $7770 \AA$ (O-triplet), and provided a resolving power of about 30000 and 22000 , respectively. A hollow-cathode ThAr lamp produced a reference spectrum of comparison lines with a FWHM of about 2 pixels. A tungsten projection lamp mounted in front of the entrance slit provided flat-fielding.

Additional high signal-to-noise ratio spectra were also obtained during several observing runs in 2001 and 2002 with the Sandiford Cassegrain Echelle Spectrograph (McCarthy et al. 1993) on the 2.1-m telescope at McDonald Observatory, USA. 
Those spectra covered continuously a wavelength range from about 4800 to $7000 \AA$ with a resolving power of about 60000 . Each night the spectrum of a broad-lined B star was obtained with a signal-to-noise ratio that exceeded those of the programme stars by a factor of several, to enable corrections for telluric lines where necessary.

By using $\operatorname{IRAF}^{1}$ we processed the CCD images, subtracted scattered light, extracted echelle orders, and performed wavelength calibrations and continuum normalization. Equivalent widths were measured from the normalized spectra by adopting a Gaussian approximation to the line profile.

Photometric data were taken from the General Catalogue of Photometric Data $\left(\mathrm{GCPD}^{2}\right)$. Where possible, averaged and weighted mean values were used throughout. To derive the effective temperatures and surface gravities, we employed the following calibrations for the individual photometric systems:

- Johnson $U B V$ : Napiwotzki et al. (1993);

- Strömgren uvbyß: Moon \& Dworetsky (1985); Napiwotzki et al. (1993);

- Geneva 7-colour: Kobi \& North (1990); Künzli et al. (1999).

When parameters from several different systems and calibrations were derived, a simple average and standard deviation was calculated; these values are listed in Table 1 . The average uncertainties were $\sigma \overline{T_{\text {eff }}}=63(23) \mathrm{K}$ and $\sigma \overline{\log g}=0.12(6)$ dex. No significant outliers were detected, so all the photometric measurements are intrinsically consistent.

To compute model atmospheres for the target stars, we employed the LLMODELS stellar model-atmosphere code (Shulyak et al. 2004). Local thermodynamical equilibrium (LTE) and plane-parallel geometry were assumed for all the calculations. We used the VALD database (Piskunov et al. 1995; Kupka et al. 1999; Ryabchikova et al. 1999) as a source of atomic line parameters for the opacity calculations and abundance determinations. We also assumed a universal microturbulent velocity $\left(v_{\text {mic }}\right)$ of $2.0 \mathrm{~km} \mathrm{~s}^{-1}$ (Landstreet et al. 2009); this choice was confirmed by our analysis of Fe I lines for the few stars for which our spectra included enough lines that were both weak and strong and could thus support a reliable determination of $v_{\text {mic }}$.

We derived the LTE abundances of the low $v \sin i$ stars $\left(v \sin i \leq 30 \mathrm{~km} \mathrm{~s}^{-1}\right.$ ) by analysing measured equivalent widths with a modified version (Tsymbal 1996) of the WIDTH9 code (Kurucz 1993). For the faster rotators we derived LTE abundances by fitting synthetic spectra that had been calculated with SYNTH3 (Kochukhov 2007). The tools and methodology adopted were the same as those described in Fossati et al. (2007). For solar abundances we used the values from Grevesse et al. (1996) in order to be compatible with the abundances published for the $\lambda$ Bootis stars by Paunzen et al. (2002). The uncertainties in the abundances were typically $\pm 0.2 \mathrm{dex}$.

The oxygen triplet at 7771-5 $\AA$ is known to be severely influenced by non-LTE (NLTE) effects (Paunzen et al. 1999). We therefore used the corrections given by Sitnova et al. (2013) for $\log g=4.0$ (see their Fig. 5) as they are valid for main-sequence stars. We applied the NLTE corrections according to the effective temperatures listed in Table 1 . None of the other lines was significantly affected by NLTE. In the case of carbon, for instance, NLTE effects for the lines that we observed are below 0.1 dex

\footnotetext{
1 IRAF is distributed by the National Optical Astronomy Observatories, which are operated by the Association of Universities for Research in Astronomy, Inc., under cooperative agreement with the National Science Foundation.

2 http://obswww. unige.ch/gcpd/
}

(Paunzen et al. 1999). The abundances thus derived are listed in Table 2.

We checked our results against those from the GenevaCopenhagen survey (Holmberg et al. 2009) and the PASTEL catalogue (Soubiran et al. 2010). The first includes kinematic data, effective temperature and absolute magnitudes, whereas the second includes effective temperature, surface gravity and $[\mathrm{Fe} / \mathrm{H}]$. The differences between the values are listed in Table 3 . We found excellent agreement between all the parameters with those listed in the literature, thus strengthening confidence in our reduction, calibration and analysis methods.

The element abundances of superficially normal stars were taken from Adelman (1991), Adelman et al. (1991), Adelman (1994), Adelman (1996), Adelman et al. (1997), Caliskan \& Adelman (1997), Hill \& Landstreet (1993), Hill (1995), Takeda et al. (2009), Varenne \& Monier (1999). The selected objects cover the complete range of $T_{\text {eff }}$ and $\log g$ in the other two samples. Values for the $\lambda$ Bootis stars were taken from Paunzen et al. (2002, Table 1 therein). We checked the literature, but did not find reports of any additional light-element abundances for the stars listed there.

\section{Discussion}

There is considerable confusion in the literature over the definitions and origins of weak-lined F-type stars on the one hand and ip II stars on the other, and this question needs further discussion.

\subsection{The weak-lined F-type stars and the ip II stars}

We first summarize the results that have already been published, and then analyse the available photometric and kinematic data.

\subsubsection{Historical facts}

Baade's original concept of a stellar "population" in the Milky Way was based on a disk (I) and a halo (II) population. The concept later evolved into a scheme with subdivisions. In one such subdivision, the old metal-weak Population II was divided into halo (extreme) and ip II objects. The latter was comprised of objects that had a velocity dispersion, a chemical composition, and a concentration towards the Galactic plane that was intermediate between those of the halo and the disk populations. The ip II population was later proposed in order to explain and fit both the global and the local Galactic kinematics. Robin \& Crézé (1986) estimated that about $1.5 \%$ of all stars in the solar neighbourhood are ip II objects.

However, Strömgren (1964) and Blauuw (1965) had already pointed out that, owing to a steady transition between the populations and also to insufficient precision in their ages, no clear distinction between the groups could be made. Strömgren (1966) therefore proposed using the $u v b y \beta$ photometric system, as it would be more illuminating. The $\delta m_{1}$ index, for instance, was used to distinguish solar from underabundant objects in the spectral-type domain between early F and mid G. Olsen (1979) listed the following boxes for cool ip II type stars (i.e. later than F4):

$$
\begin{aligned}
+0.270<(b-y) & <+0.412 \\
+0.045<\delta m_{1} & <+0.100 \\
-0.045<\delta c_{1} & <+0.280
\end{aligned}
$$

For the hotter stars $\left(+0.043<(b-y)<+0.270\right.$ and $\left.+0.045<\delta m_{1}\right)$ Strömgren found overlaps with "classical" $\lambda$ Bootis stars and 
Table 2. Element abundances for our sample of stars, relative to the solar values given by Grevesse et al. (1996).

\begin{tabular}{|c|c|c|c|c|c|c|c|c|c|c|}
\hline $\mathrm{HD}$ & $\mathrm{C}_{\mathrm{I}}$ & Si I & Ti I & CrI & $\mathrm{Fe} \mathrm{I}$ & NiI & $\mathrm{KI}_{\mathrm{I}}$ & $\mathrm{Ca} \mathrm{I}$ & O I (LTE) & O I (NLTE) \\
\hline 693 & -0.10 & +0.12 & -0.25 & -0.20 & -0.36 & -0.38 & & & & \\
\hline 3229 & -0.02 & +0.02 & -0.19 & -0.03 & -0.23 & -0.31 & & & & \\
\hline 4813 & +0.17 & & -0.32 & -0.06 & -0.17 & -0.23 & & & & \\
\hline 7476 & -0.05 & -0.08 & -0.11 & +0.05 & -0.19 & -0.28 & & & & \\
\hline 9919 & -0.52 & +0.06 & & & -0.11 & +0.78 & +0.78 & +0.33 & +0.75 & +0.09 \\
\hline 12414 & -0.23 & -0.14 & -0.25 & -0.22 & -0.34 & -0.40 & -0.12 & -0.24 & +0.14 & -0.38 \\
\hline 13555 & -0.15 & -0.12 & -0.06 & +0.08 & -0.20 & -0.27 & +0.58 & +0.05 & +0.19 & -0.37 \\
\hline 16626 & -0.29 & -0.44 & -0.14 & -0.18 & -0.37 & -0.34 & +0.16 & +0.19 & +0.17 & -0.42 \\
\hline 17948 & -0.29 & -0.21 & & & -0.31 & -0.50 & -0.05 & -0.15 & +0.17 & -0.40 \\
\hline 24740 & -0.14 & -0.04 & & & -0.09 & -0.27 & +1.10 & +0.69 & +1.12 & +0.44 \\
\hline 25457 & -0.02 & +0.15 & & & +0.20 & -0.14 & +0.47 & +0.52 & +0.33 & -0.18 \\
\hline 26015 & +0.23 & +0.12 & & & +0.13 & +0.04 & +0.88 & +0.80 & +0.86 & +0.23 \\
\hline 27397 & +0.03 & -0.36 & & & -0.06 & +1.49 & +0.44 & +1.14 & +1.06 & +0.28 \\
\hline 27524 & & +0.11 & & & +0.18 & +0.59 & +0.55 & & +0.61 & +0.02 \\
\hline 27808 & & +0.26 & & & +0.23 & +0.11 & +0.59 & & +0.34 & -0.16 \\
\hline 29391 & & +0.00 & & & +0.44 & +0.46 & +0.75 & & +1.05 & +0.27 \\
\hline 33256 & & -0.14 & & & -0.27 & -0.35 & +0.40 & & +0.19 & -0.35 \\
\hline 65123 & -0.09 & +0.02 & & & -0.01 & -0.16 & +0.27 & +0.30 & +0.12 & -0.40 \\
\hline 69897 & -0.15 & -0.15 & & & -0.21 & -0.33 & +0.06 & +0.08 & +0.01 & -0.52 \\
\hline 110093 & & -0.33 & & & -0.08 & +0.45 & & +0.41 & +0.78 & -0.03 \\
\hline 114642 & -0.16 & & & & -0.04 & & & +0.07 & +0.46 & -0.08 \\
\hline 125111 & -0.44 & -0.15 & & & -0.35 & -0.53 & +0.21 & -0.02 & +0.42 & -0.20 \\
\hline 125276 & -0.42 & & & & -0.49 & & & +0.65 & & \\
\hline 128167 & -0.54 & -0.44 & & & -0.44 & -0.56 & -0.01 & -0.20 & +0.02 & -0.61 \\
\hline 135208 & -0.43 & & & & -0.37 & & & -0.18 & & \\
\hline 137006 & -0.13 & +0.13 & & & -0.27 & +1.08 & -0.24 & +1.06 & +0.96 & +0.15 \\
\hline 147449 & -0.05 & +0.21 & & & -0.10 & +0.68 & -0.03 & & +0.91 & +0.21 \\
\hline 157950 & -0.54 & -0.69 & & & -0.48 & -1.71 & & -0.01 & +0.83 & +0.21 \\
\hline 185124 & +0.39 & & & & +0.15 & & & +0.44 & & \\
\hline 189712 & -0.23 & -0.43 & -0.30 & -0.27 & -0.45 & -0.49 & & & & \\
\hline 191841 & +0.03 & -0.05 & -0.19 & & -0.36 & -0.45 & & & & \\
\hline 198390 & -0.31 & -0.21 & -0.06 & -0.09 & -0.32 & -0.35 & +0.38 & -0.13 & -0.01 & -0.58 \\
\hline 199124 & -0.39 & -0.22 & & & -0.43 & -1.44 & -0.70 & & +0.95 & +0.20 \\
\hline 208362 & -0.18 & -0.50 & -0.06 & -0.66 & -0.48 & -0.61 & +0.09 & -0.07 & +0.38 & -0.25 \\
\hline 211575 & +0.23 & +0.05 & & +0.59 & +0.14 & -0.27 & +0.67 & +0.54 & +0.53 & -0.05 \\
\hline 218804 & -0.25 & +0.00 & & -0.48 & -0.07 & -0.41 & +0.67 & -0.12 & +0.27 & -0.28 \\
\hline 222368 & -0.11 & +0.02 & & & -0.36 & -0.23 & +0.52 & -0.30 & +0.19 & -0.31 \\
\hline 223346 & -0.15 & -0.42 & & & -0.20 & -0.21 & +0.47 & +0.16 & +0.32 & -0.21 \\
\hline
\end{tabular}

Notes. Typical uncertainties are \pm 0.2 dex.

Table 3. Differences between our derived values for the target sample and those in the Geneva-Copenhagen survey (GCS, Holmberg et al. 2009) and the PASTEL catalogue (Soubiran et al. 2010).

\begin{tabular}{lcccc}
\hline \hline Parameter & GCS & \multicolumn{2}{c}{ PASTEL } \\
& mean & $\mathrm{N}$ & mean & $\mathrm{N}$ \\
\hline$\Delta T_{\text {eff }}[\mathrm{K}]$ & $+44 \pm 54$ & 33 & $+8 \pm 78$ & 35 \\
$\Delta \log g[\mathrm{dex}]$ & & & $+0.03 \pm 0.14$ & 22 \\
$\Delta M_{\mathrm{V}}[\mathrm{mag}]$ & $-0.01 \pm 0.08$ & 33 & & \\
$\Delta[\mathrm{Fe} / \mathrm{H}][\mathrm{dex}]$ & & & $+0.05 \pm 0.14$ & 22 \\
$\Delta U\left[\mathrm{~km} \mathrm{~s}^{-1}\right]$ & $+0.33 \pm 1.32$ & 33 & & \\
$\Delta V\left[\mathrm{~km} \mathrm{~s}^{-1}\right]$ & $-0.61 \pm 2.11$ & 33 & & \\
$\Delta W\left[\mathrm{~km} \mathrm{~s}^{-1}\right]$ & $+0.51 \pm 1.48$ & 33 & & \\
\hline
\end{tabular}

with field horizontal-branch stars which could then be separated easily by their $c_{1}$ values.

Gray (1989) obtained spectra at a classification dispersion $\left(67 \AA \mathrm{mm}^{-1}\right)$ of a sample of metal-weak stars that were selected according to the above criteria. He established metalweak standards for that spectral range so as to extend the
Yerkes MK system, and labelled his sample "intermediate Population II-type stars".

Independently, Jaschek et al. (1989) conducted a survey of metal-weak F-type stars, and also used spectra with a classification dispersion $\left(80 \AA \mathrm{mm}^{-1}\right)$. Both Jaschek et al. and Gray employed the strengths of the Balmer lines, the G-band $(\mathrm{CH})$, and metallic lines, but Jaschek et al. labelled their sample of metal-weak F-type stars simply "F-weak type stars". Hauck et al. (1991) subsequently investigated the same sample as Jaschek et al. using the Geneva photometric system; the metallicitysensitive indices that calibrate $[\mathrm{Fe} / \mathrm{H}]$ are the same as those in the Strömgren system.

In this paper we have therefore adopted the expression ip II group/stars to describe stars listed in the literature as members of either the ip II or the F-weak group.

\subsubsection{Photometric and kinematic data}

We employed the Strömgren $u v b y \beta$ system search for similarities between the two groups. Figure 1 plots unreddened indices $\left[m_{1}\right],\left[c_{1}\right]$, and $\beta$ for $\lambda$ Bootis stars (Paunzen et al. 2002), F-weak 
E. Paunzen et al.: $\lambda$ Bootis stars and intermediate Population II type stars

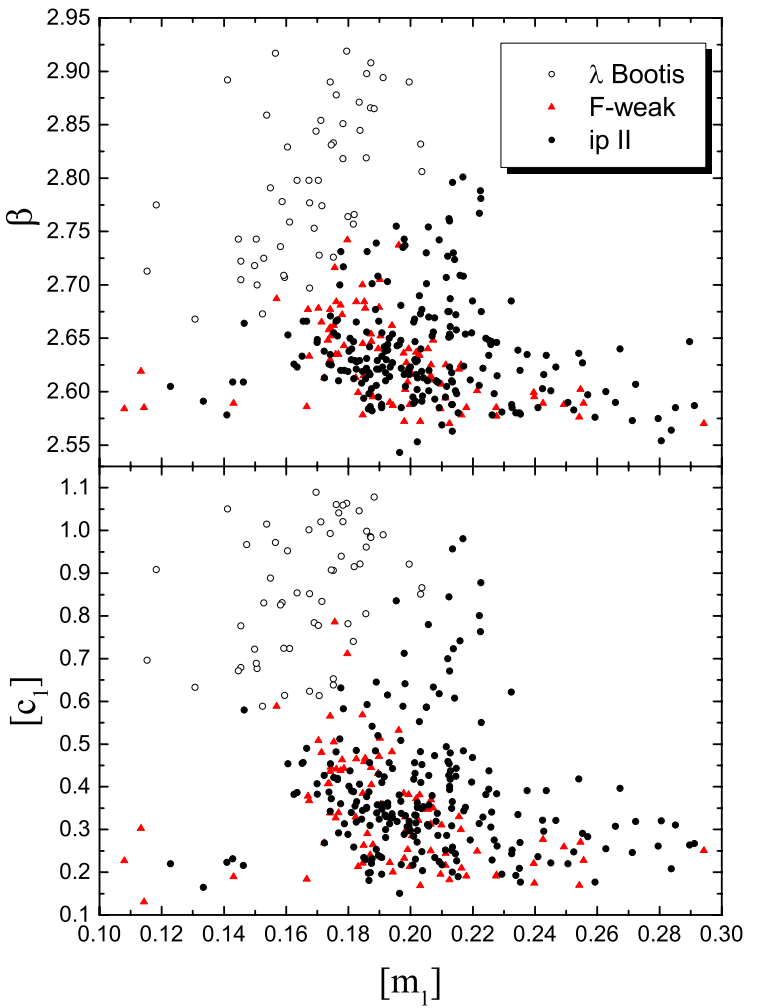

Fig. 1. Photometric plots of the unreddened indices $\left[m_{1}\right],\left[c_{1}\right]$, and $\beta$ for $\lambda$ Bootis (open circles), F-weak (red triangles), and ip II (black triangles) stars.

(Jaschek et al. 1989) stars, and ip II stars (Gray 1989). To derive reddening-free parameters we used the definition originally introduced by Strömgren (1966):

$$
\begin{aligned}
{\left[m_{1}\right] } & =m_{1}+0.18(b-y), \\
{\left[c_{1}\right] } & =c_{1}-0.20(b-y) .
\end{aligned}
$$

In general, $\beta$ is temperature sensitive, $\left[m_{1}\right]$ correlates with metallicity, and $\left[c_{1}\right]$ is correlated with surface gravity and thus with the age of a star for a given spectral type. As the figure indicates, there is no clear separation between the two groups.

That result from the photometric data was supported by kinematical data which were computed from proper motions (from the HIPPARCos catalogue) and mean radial velocities (from the literature) as described in Johnson \& Soderblom (1987). A righthanded coordinate system for $U, V$, and $W$ was used, so the velocities are positive in the directions of the Galactic centre, Galactic rotation and the North Galactic Pole. The standard solar motion in that system is in units of $\left[\mathrm{km} \mathrm{s}^{-1}\right](+10.4,+14.8$, +7.3 ) according to Mihalas \& Routly (1968). For our analysis we adopted the $V$, and the quantity $Q=\sqrt{U^{2}+W^{2}}$ which is a measure of the kinematic energy other than that associated with rotation. Since all velocities are heliocentric, stars with $V>-40 \mathrm{~km} \mathrm{~s}^{-1}$ and $Q<65 \mathrm{~km} \mathrm{~s}^{-1}$ are probable disk members (Caloi et al. 1999). Figure 2 plots $Q=\sqrt{U^{2}+W^{2}}$ against $V$. There is no distinction between the two groups in that diagram.

The following conclusion can be drawn from this analysis:

- There are no obvious differences between the sample chosen by Gray (1989) and the one selected by Jaschek et al. (1989). This means that ip II and F-weak type stars are simply names for the same group of stars.

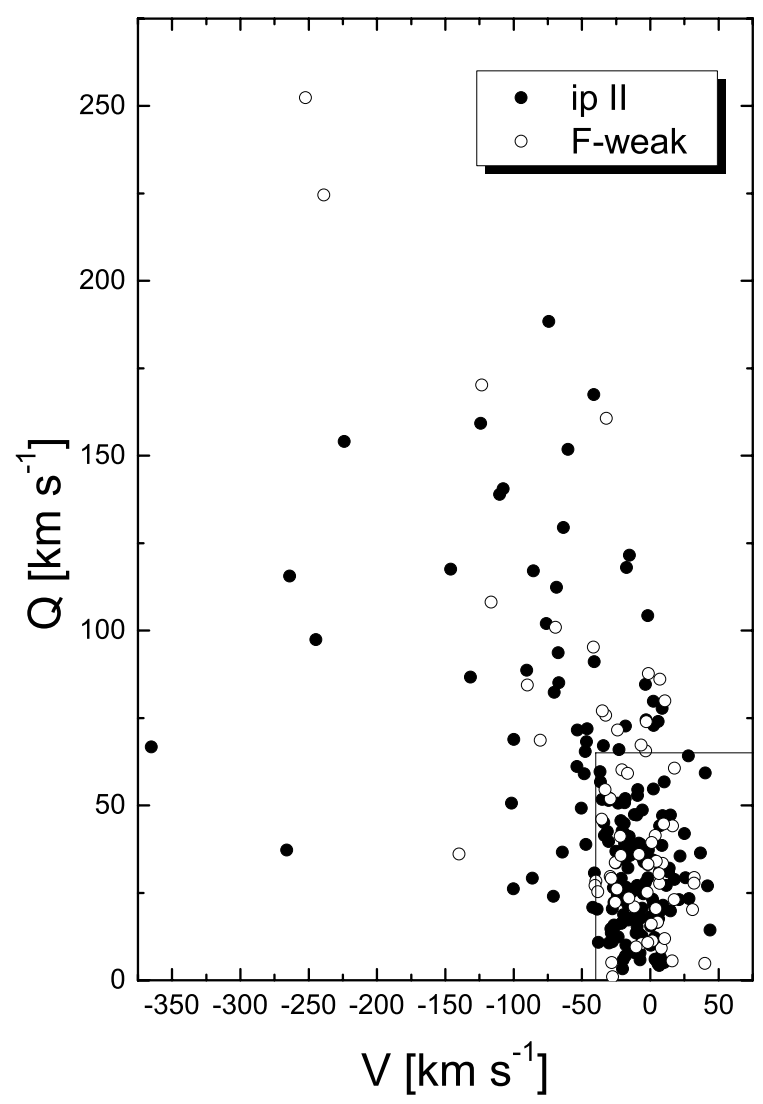

Fig. 2. $Q=\sqrt{U^{2}+W^{2}}$ versus $V$ diagram for ip II (filled circles) and Fweak (open circles) stars. The plot is a measure of the kinematic energy not associated with rotation. The two groups are not distinguishable. The rectangle marked is the empirical limit for disk stars according to Caloi et al. (1999).

\subsection{The groups of $\lambda$ Bootis and ip II stars}

One of the most important features that distinguishes $\lambda$ Bootis from other stellar groups is their unusual abundance patterns. Although the light elements have solar abundances, the heavier elements are overabundant but nearly to the same extent. This means that, for example, silicon shows more or less the same amount of overabundance as iron. We therefore investigated these abundance characteristics shown by our selected sample. Figure 3 shows the results for three different combinations: $[\mathrm{Fe} / \mathrm{C}]$ versus $[\mathrm{Fe} / \mathrm{H}],[\mathrm{Fe} / \mathrm{O}]$ versus $[\mathrm{Fe} / \mathrm{H}]$, and $[\mathrm{Fe} / \mathrm{H}]$ versus $[\mathrm{Ca} / \mathrm{H}]$. In all three diagrams, the ip II stars are located among the normal non-peculiar objects, the $\lambda$ Bootis stars are clearly separated from them, and the light elements are offset by -0.5 dex. The behaviour of the heavier elements was then examined for possible correlations between the samples in respect of $[\mathrm{Fe} / \mathrm{H}]$ versus $[\mathrm{Ca} / \mathrm{H}]$. For the normal, ip II, and $\lambda$ Bootis samples (see Fig. 3) we derived

$$
\begin{aligned}
& {[\mathrm{Fe} / \mathrm{H}]=-0.01(2)+0.32(9)[\mathrm{Ca} / \mathrm{H}], N=95,} \\
& {[\mathrm{Fe} / \mathrm{H}]=-0.25(4)+0.26(9)[\mathrm{Ca} / \mathrm{H}], N=26,} \\
& {[\mathrm{Fe} / \mathrm{H}]=-0.68(13)+0.40(11)[\mathrm{Ca} / \mathrm{H}], N=24,} \\
& {[\mathrm{Fe} / \mathrm{H}]=-0.32(11)+0.83(12)[\mathrm{Ca} / \mathrm{H}], N=23 .}
\end{aligned}
$$

here $N$ denotes the number of available individual abundances for each star group.

The last correlation did not include the outlier (HD 91130) which has a calcium abundance of -3.0 dex. The first two samples show a slope of about 0.30 whereas the $\lambda$ Bootis stars 

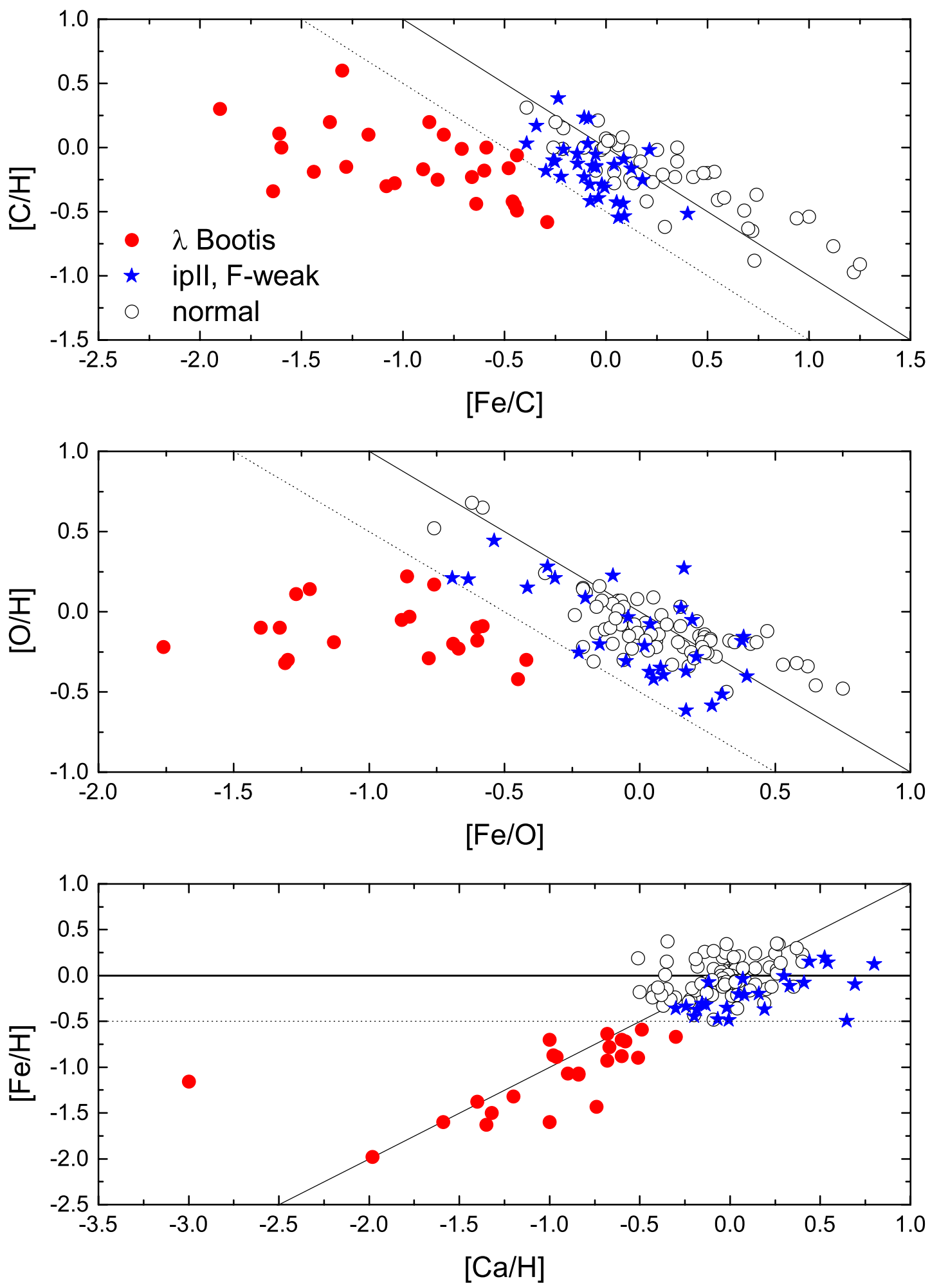

Fig. 3. Different element abundances for the star groups investigated. The $\lambda$ Bootis stars are clearly separated.

show almost no slope. The ip II sample overlaps the normal stars across a wide range of abundances.

From this analysis, we define a $\lambda$ Bootis star on the basis of elemental abundances if

1. $[\mathrm{C} / \mathrm{H}]<-0.50-[\mathrm{Fe} / \mathrm{C}]$,

2. $[\mathrm{O} / \mathrm{H}]<-0.50-[\mathrm{Fe} / \mathrm{O}]$,

3. $[\mathrm{Fe} / \mathrm{H}]<-0.50$,

4. Heavy elements scale with $[\mathrm{Fe} / \mathrm{H}]$.
This definition separates the ip II stars from $\lambda$ Bootis stars.

We also analysed the photometric and kinematic data of both groups. (see Sect. 3.1). Figure 1 plots the unreddened indices $\left[m_{1}\right],\left[c_{1}\right]$, and $\beta$. The $\lambda$ Bootis stars scarcely overlap at all with the ip II objects. That characteristic could become an additional criterion for distinguishing both groups.

Figure 4 shows the kinematic data for the $\lambda$ Bootis stars and the ip II (Table 1) sample. The two outliers (HD 6870 and 
E. Paunzen et al.: $\lambda$ Bootis stars and intermediate Population II type stars

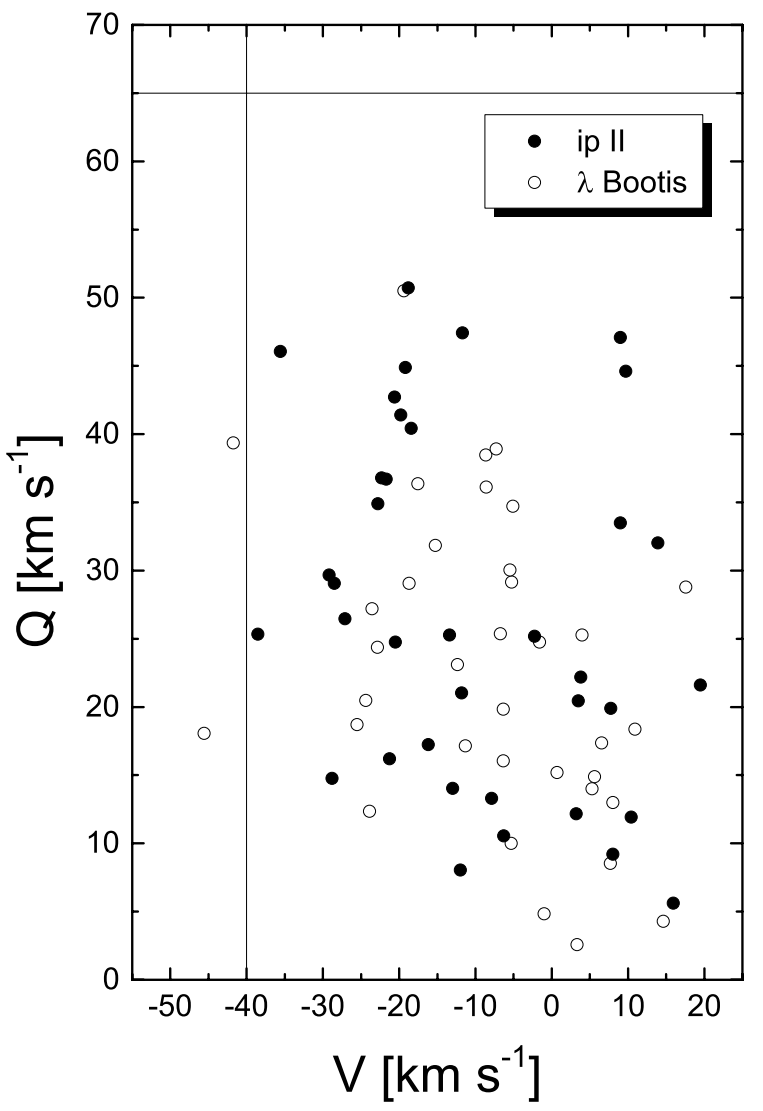

Fig. 4. $Q=\sqrt{U^{2}+W^{2}}$ plotted against $V$. $\lambda$ Bootis stars are shown as open circles, ip II stars as filled circles. The horizontal and vertical lines are the empirical limits for disk stars according to Caloi et al. (1999).

Table 4. [Z] values for the $\lambda$ Bootis stars (from Paunzen et al. 2002) using individual elemental abundances.

\begin{tabular}{lc|lc}
\hline \hline Star & {$[Z]$} & Star & {$[Z]$} \\
\hline HD 319 & 0.018 & HD 120500 & 0.013 \\
HD 11413 & 0.010 & HD 125162 & 0.007 \\
HD 15165 & 0.008 & HD 142703 & 0.007 \\
HD 31295 & 0.007 & HD 168740 & 0.010 \\
HD 74873 & 0.024 & HD 170680 & 0.012 \\
HD 75654 & 0.014 & HD 183324 & 0.011 \\
HD 84123 & 0.009 & HD 192640 & 0.007 \\
HD 91130 & 0.018 & HD 193256 & 0.008 \\
HD 101108 & 0.016 & HD 193281 & 0.012 \\
HD 106223 & 0.024 & HD 198160 & 0.010 \\
HD 107233 & 0.006 & HD 204041 & 0.006 \\
HD 110411 & 0.017 & HD 210111 & 0.008 \\
HD 111604 & 0.007 & HD 221756 & 0.018 \\
\hline
\end{tabular}

Notes. The absolute solar values were taken from Grevesse et al. (1996); $[Z]_{\odot}=0.017$.

HD 84123) were already been discussed in Paunzen et al. (2002). Their $U$ and $W$ velocities qualify them as being Population I objects. In respect of our total sample, we can state that they all belong kinematically to the Galactic disk. We therefore conclude that there is no distinction between the vast majority of the members of either group on the basis of their kinematic data.

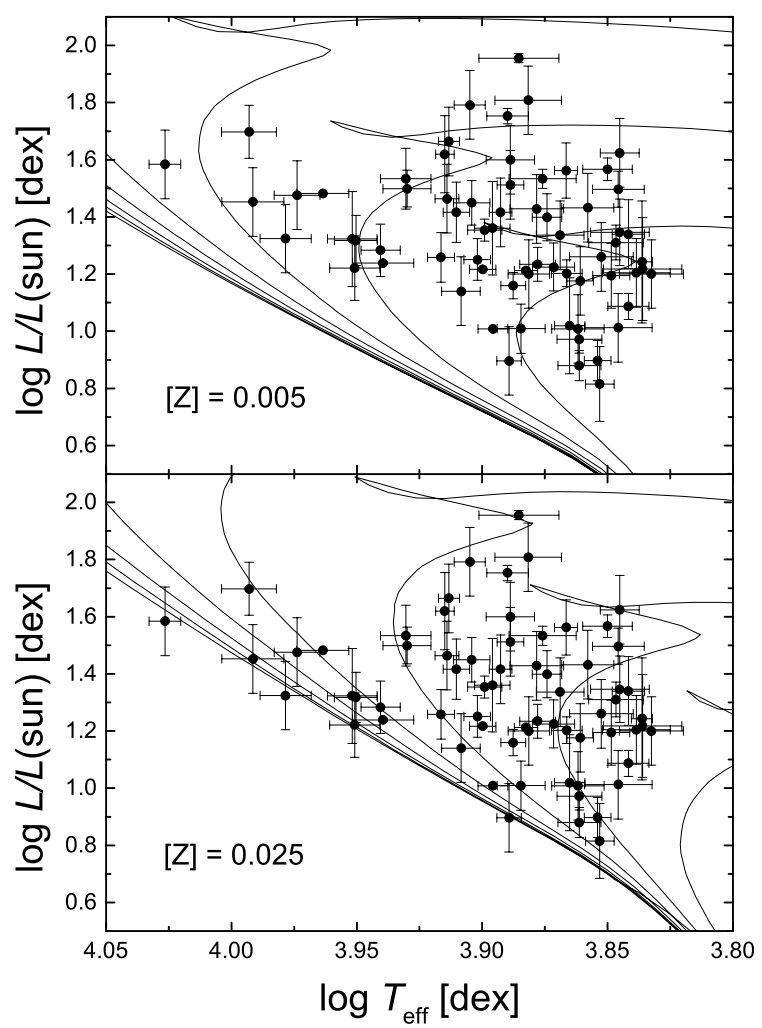

Fig. 5. $\lambda$ Boo stars in the Hertzsprung-Russell diagram, with isochrones from Bressan et al. (2012) for the whole range of $[Z]$ values listed in Table 4.

\subsection{Evolutionary status and metallicity}

In order to investigate the possibility of an intrinsic chemical peculiarity in the $\lambda$ Bootis stars, we calculated individual $[Z]$ values for 26 objects whose abundances are listed in Table 5 of Paunzen et al. (2002). The absolute mass fraction abundances $(\chi)$ were taken from Grevesse et al. (1996); $[Z]_{\odot}=0.017$. Where light-element abundances were not known, the value for carbon was used; the value for iron was adopted as representative of Fe-peak elements. In total, we included all elements for which $\chi>10^{-8}$. Table 4 lists our results for the sample. The light elements $(\mathrm{C}, \mathrm{N}, \mathrm{O}$, and $\mathrm{S})$ distribute the most, in absolute numbers, for $[Z]$. Therefore, two stars (HD 74873 and HD 106223) even have absolute overabundances with $[Z]=0.024$, while all other objects have values as low as 0.006 . As a next step, we examined the relationship between metallicity and ages by selecting isochrones from (from Bressan et al. 2012) for the corresponding metallicity values. Figure 5 plots the Hertzsprung-Russell diagram for the corresponding range of $[Z]$. Several stars lie beyond the terminal-age main sequence (luminosity class IV), but none close to the zero-age main-sequence. The complete metallicity range is compatible with the observed data, so (within the errors) no objects is located below the zero-age main sequence (which from an astrophysical standpoint would not be possible).

\section{Conclusions and outlook}

We investigated a possible connection between the $\lambda$ Bootis stars and intermediate Population II, F-weak stars on the basis of elemental abundances and kinematic data. It tackled the important questions whether the $\lambda$ Boo stars are intrinsically metal-weak, and thus belong to the intermediate Population II, or whether their ususual abundances are restricted to the outer layers of the 
atmosphere. Recent results from an asteroseismologic study suggest the former. If so, the $\lambda$ Bootis stars become even more interesting, especially those in which accretion disks have been detected.

Our analysis of $38 \mathrm{ip} \mathrm{II/F-weak} \mathrm{stars} \mathrm{was} \mathrm{based} \mathrm{on} \mathrm{detailed}$ element abundances, including the light elements carbon and oxygen, and kinematic data from the HIPPARCos catalogue.

First we investigated whether the ip II and F-weak stars are separate groups or whether the names are merely synonyms for identical objects. From photometric, spectroscopic and kinematic data, we conclude that the two groups are indeed identical.

We then compared the elemental abundances of normal, $\lambda$ Bootis, and ip II stars. The ip II group overlaps with the normal stars, but is completely separated from the $\lambda$ Bootis objects. We, therefore, defined four constraints regarding the discrimination of both groups. From the kinematic data, no similar differentiation can be drawn.

Finally, we calculated the overall $[Z]$ values for the $\lambda$ Bootis stars and plotted them, along with corresponding isochrones, in the Hertzsprung-Russells diagram. Using metal-poor isochrones would shift several stars beyond the terminal-age main sequence.

As independent tests, we propose that asteroseismological studies (Casas et al. 2009) be made of pulsating members, and that spectroscopic binary systems (Iliev et al. 2002) containing $\lambda$ Bootis-type components be investigated. One very interesting star in that respect is HD 210111, which is a $\delta$ Scuti pulsator in a spectroscopic binary system.

Another interesting stellar system is the eclipsing binary HR 854 ( $\tau$ Persei). The primary is a late G-type giant, and the secondary could belong to the $\lambda$ Bootis group (Griffin et al. 1992). Its K-line type is earlier than derived from the hydrogen lines (Ginestet \& Carquillat 2002). Therefore, this system is an excellent test for fitting isochrones of different metallicities.

Acknowledgements. This project is financed by the SoMoPro II programme (3SGA5916). The research leading to these results has acquired a financial grant from the People Programme (Marie Curie action) of the Seventh Framework Programme of EU according to the REA Grant Agreement No. 291782. The research is further co-financed by the South-Moravian Region. It was also supported by the grant 7AMB14AT015, the financial contributions of the Austrian Agency for International Cooperation in Education and Research (BG-03/2013 and CZ-09/2014), NSF grant 01/7 - DNTS AT, and the Austrian Research Fund via the project FWF P22691-N16. We thank our referee, Elizabeth Griffin, for her efforts to significantly improve this paper. This work reflects only the author's views and the European Union is not liable for any use that may be made of the information contained therein.

\section{References}

Adelman, S. J. 1991, MNRAS, 252, 116

Adelman, S. J. 1994, MNRAS, 271, 355

Adelman, S. J. 1996, MNRAS, 280, 130

Adelman, S. J., Bolcal, C., Hill, G., \& Kocer, D. 1991, MNRAS, 252, 329

Adelman, S. J., Caliskan, H., Kocer, D., \& Bolcal, C. 1997, MNRAS, 288, 470

Blauuw, A. 1965, in Galactic Structure, eds. A. Blauuw, \& M. Schmidt (Chicago:

University of Chicago Press), 435
Booth, M., Kennedy, G., Sibthorpe, B. et al. 2013, MNRAS, 428, 1263

Breger, M., Beck, P., Lenz, P., et al. 2006, A\&A, 455, 673

Bressan, A., Marigo, P., Girardi, L., et al. 2012, MNRAS, 427, 127

Caliskan, H., \& Adelman, S. J. 1997, MNRAS, 288, 501

Caloi, V., Cardini, D., D’Antona, F., et al. 1999, A\&A, 351, 925

Casas, R., Moya, A., Suárez, J. C., et al. 2009, ApJ, 697, 522

Fossati, L., Bagnulo, S., Monier, R., et al. 2007, A\&A, 476, 911

Ginestet, N., \& Carquillat, J. M. 2002, ApJS, 143, 513

Gray, R. O. 1989, AJ, 98, 1049

Grevesse, N., Noels, A., \& Sauval, A. J. 1996, in Cosmic Abundances, eds. S. S. Holt, \& G. Sonneborn, ASP Conf. Ser., 99, 117

Griffin, R. E. M., Schroder, K. P., Misch, A., \& Griffin, R. F. 1992, A\&A, 254 289

Hauck, B., Jaschek, C., Jaschek, M., \& Andrillat Y. 1991, A\&A, 252, 260

Hill, G. M. 1995, A\&A, 294, 536

Hill, G. M., \& Landstreet, J. D. 1993, A\&A, 276, 142

Holmberg, J., Nordström, B., \& Andersen, J. 2009, A\&A, 501, 941

Iliev, I. Kh., Paunzen, E., Barzova, I. S., et al. 2002, A\&A, 381, 914

Jaschek, M., Andrillat, Y., \& Jaschek, C. 1989, A\&A, 218, 180

Johnson, D. R. H., \& Soderblom, D. R. 1987, AJ, 93, 864

Kamp, I., \& Paunzen, E. 2002, MNRAS, 335, L45

Kobi, D., \& North, P. 1990, A\&AS, 85, 999

Kochukhov, O. 2007, Spectrum synthesis for magnetic, chemically stratified stellar atmospheres, in Magnetic Stars 2006, eds I. I. Romanyuk, D. O. Kudryavtsev, O. M. Neizvestnaya, \& V. M. Shapoval, 109, 118

Künzli, M., North, P., Kurucz, R. L., \& Nicolet, B. 1997, A\&AS, 122, 51

Kupka, F., Piskunov, N., Ryabchikova, T. A., Stempels, H. C., \& Weiss, W. W. 1999, A\&AS, 138, 119

Kurucz, R. 1993, ATLAS9: Stellar Atmosphere Programs and $2 \mathrm{~km} \mathrm{~s}^{-1}$ grid. Kurucz CD-ROM No. 13 (Cambridge: Smithsonian Astrophysical Observatory)

Landstreet, J. D., Kupka, F., Ford, H. A., et al. 2009, A\&A, 503, 973

Martinez-Galarza, J. R., Kamp, I., Su, K. Y. L., et al. 2009, AJ, 694, 165

McCarthy, J. K., Sandiford, B. A., Boyd, D., \& Booth, J. 1993, PASP, 105, 881

Mihalas, D., \& Routly, P. M. 1968, Galactic Astronomy, Freeman \& Co., San Francisco, 101

Moon, T. T., \& Dworetsky, M. M. 1985, MNRAS, 217, 305

Napiwotzki, R., Schoenberner, D., \& Wenske, V. 1993, A\&A, 268, 653

Netopil, M., Paunzen, E., Maitzen, H. M., North, P., \& Hubrig, S. 2008, A\&A, 491, 545

Olsen, E. H. 1979, A\&AS, 37, 367

Paunzen, E., Kamp, I., Iliev, I. Kh., et al. 1999, A\&A, 345, 597

Paunzen, E., Iliev, I. Kh., Kamp, I., \& Barzova, I. 2002, MNRAS, 336, 1030

Paunzen, E., Kamp, I., Weiss, W. W., \& Wiesemeyer, H. 2003, A\&A, 404, 579

Paunzen, E., Heiter, U., Fraga, L., \& Pintado, O. 2012, MNRAS, 419, 3604

Piskunov, N. E., Kupka, F., Ryabchikova, T. A., Weiss, W. W., \& Jeffery, C. S. 1995, A\&AS, 112, 525

Robin, A., \& Crézé, M. 1986, A\&A, 157, 71

Ryabchikova, T. A., Piskunov, N. E., Stempels, H. C., Kupka, F., \& Weiss, W. W. 1999, Phis. Scr. T, 83, 162

Shulyak, D., Tsymbal, V., Ryabchikova, T., Stütz, Ch., \& Weiss, W. W. 2004, A\&A, 428, 993

Sitnova, T. M., Mashonkina, L. I., \& Ryabchikova, T. A. 2013, Astron. Lett., 39, 126

Soubiran, C., Le Campion, J.-F., Cayrel de Strobel, G., \& Caillo, A. 2010, A\&A, 515, A111

Strömgren, B. 1964, Astrophys. Norv., 9, 333

Strömgren, B. 1966, ARA\&A, 4, 433

Takeda, Y., Kang, D.-I., Han, I., Lee, B.-C., \& Kim, K.-M. 2009, PASJ, 61, 1165

Tsymbal, V. V. 1996, in Model Atmospheres and Spectral Synthesis, eds. S. J. Adelman, F. Kupka, \& W. W., Weiss, ASP Conf. Ser., 108, 198

van Leeuwen, F. 2009, A\&A, 497, 209

Varenne, O., \& Monier, R. 1999, A\&A, 351, 247

Venn, K. A., \& Lambert, D. L. 1990, ApJ, 363, 234 